Monte Carlo Treatment Planning for Molecular Targeted Radiotherapy within the MINERVA System

Joerg Lehmann, Christine Hartmann Siantar, Daniel E. Wessol, Charles A. Wemple, David Nigg, Josh Cogliati, Tom Daly, Marie-Anne Descalle, Terry Flickinger, David Pletcher, Gerald DeNardo

September 23, 2004

Current Topics in Monte Carlo Treatment Planning Advanced Workshop Montreal, Canada May 3, 2004 through May 5, 2004 
This document was prepared as an account of work sponsored by an agency of the United States Government. Neither the United States Government nor the University of California nor any of their employees, makes any warranty, express or implied, or assumes any legal liability or responsibility for the accuracy, completeness, or usefulness of any information, apparatus, product, or process disclosed, or represents that its use would not infringe privately owned rights. Reference herein to any specific commercial product, process, or service by trade name, trademark, manufacturer, or otherwise, does not necessarily constitute or imply its endorsement, recommendation, or favoring by the United States Government or the University of California. The views and opinions of authors expressed herein do not necessarily state or reflect those of the United States Government or the University of California, and shall not be used for advertising or product endorsement purposes. 


\title{
Monte Carlo Treatment Planning for Molecular Targeted Radiotherapy within the MINERVA system
}

\author{
Joerg Lehmann ${ }^{1,4}$, Christine Hartmann Siantar ${ }^{1,4}$, Daniel E. Wessol ${ }^{2}$, \\ Charles A. Wemple ${ }^{2}$, David Nigg ${ }^{2}$, Josh Cogliati ${ }^{3}$, Tom Daly ${ }^{1}$, Marie-Anne Descalle ${ }^{1}$, \\ Terry Flickinger ${ }^{1}$, David Pletcher ${ }^{1}$, Gerald DeNardo ${ }^{4}$ \\ ${ }^{1}$ University of California - Lawrence Livermore National Laboratory, 7000 East Ave, \\ Livermore, CA 94550, USA \\ ${ }^{2}$ Idaho National Engineering and Environmental Laboratory, P.O. Box 1625, Idaho Falls, \\ ID 83415-3885, USA \\ ${ }^{3}$ Montana State University, Department of Computer Science, Bozeman, MT 59717, \\ USA \\ ${ }^{4}$ University of California Davis School of Medicine, Sacramento, CA 95817, USA
}

Short title: Monte Carlo for Molecular Targeted Radiotherapy

Classification numbers: $87.53 . \mathrm{Na}$, 87.50.Gi, $87.58 . \mathrm{Sp}$

\begin{abstract}
The aim of this project is to extend accurate and patient-specific treatment planning to new treatment modalities, such as molecular targeted radiation therapy, incorporating previously crafted and proven Monte Carlo and deterministic computation methods. A flexible software environment is being created that allows planning radiation treatment for these new modalities and combining different forms of radiation treatment
\end{abstract}


with consideration of biological effects. The system uses common input interfaces, medical image sets for definition of patient geometry, and dose reporting protocols.

Previously, the Idaho National Engineering and Environmental Laboratory (INEEL), Montana State University (MSU), and Lawrence Livermore National Laboratory (LLNL) had accrued experience in the development and application of Monte Carlo-based, three-dimensional, computational dosimetry and treatment planning tools for radiotherapy in several specialized areas. In particular, INEEL and MSU have developed computational dosimetry systems for neutron radiotherapy and neutron capture therapy, while LLNL has developed the PEREGRINE computational system for external beam photon-electron therapy. Building on that experience, the INEEL and MSU are developing the MINERVA (Modality Inclusive Environment for Radiotherapeutic Variable Analysis) software system as a general framework for computational dosimetry and treatment planning for a variety of emerging forms of radiotherapy. In collaboration with this development, LLNL has extended its PEREGRINE code to accommodate internal sources for molecular targeted radiotherapy (MTR), and has interfaced it with the plugin architecture of MINERVA. Results from the extended PEREGRINE code have been compared to published data from other codes, and found to be in general agreement (EGS4 - 2\%, MCNP - 10\%)(Descalle et al. 2003). The code is currently being benchmarked against experimental data. The interpatient variability of the drug pharmacokinetics in MTR can only be properly accounted for by image-based, patientspecific treatment planning as has been common in external beam radiation therapy for many years. MINERVA offers 3D Monte Carlo based MTR treatment planning as its first integrated operational capability. 
The new MINERVA system will ultimately incorporate capabilities for a comprehensive list of radiation therapies. In progress are modules for external beam photon-electron therapy and Boron Neutron Capture Therapy (BNCT). Brachytherapy and Protontherapy are planned. Through the open Application Programming Interface (API) other groups can add their own modules and share them with the community.

\section{Introduction}

The MINERVA (Modality Inclusive Environment for Radiotherapeutic Variable Analysis) project intends to extend accurate and patient-specific treatment planning to new modalities, such as molecular targeted radiation therapy (MTR). Many patients who receive MTR have previously been treated with other modalities of radiation therapy, which makes the availability of biologically weighted combined plans highly desirable (Bodey et al. 2003). MINERVA offers the clinician the potential to evaluate treatment strategies consisting of composite plans of different forms of radiation treatment incorporating specific biological factors. The project is a joint effort of the Idaho National Engineering and Environmental Laboratory (INEEL), Montana State University (MSU), Lawrence Livermore National Laboratory (LLNL), and the University of California Davis, School of Medicine. MINERVA builds on accrued experience in the design of three-dimensional Monte Carlo treatment planning tools. INEEL and MSU have developed the BNCT_rtpe and SERA computational dosimetry systems for neutron radiotherapy and neutron capture therapy (Nigg et al. 1997, Nigg 2003), while LLNL has developed the PEREGRINE Monte Carlo system for external photon beam therapy (Hartmann Siantar et al. 2001). MINERVA employs an integrated, lightweight plugin 
architecture to accommodate multi-modal treatment planning using standard interface components.

The work presented here focuses on the treatment planning for MTR within MINERVA, as accurate treatment planning is important to the success of MTR treatment (DeNardo et al. 1985, DeNardo et al. 2003, Thomas 2002). For external beam radiation therapy, calculation of patient specific doses delivered to tumor and normal tissue before treatment is generally accepted as necessary. Studies conducted over several decades have shown that individualized treatment planning improves the response to the radiation treatment and decreases morbidity. The necessity to expand patient specific radiation treatment planning to radionuclide therapy has been acknowledged (Flux et al. 2002, Stabin 1999). The available knowledge of the response of specific tumor types to radiation and the relation between radiation dose and early and late tissue toxicity (Rubin 1989, Benua et al. 1962) will facilitate this effort.

In MTR, radionuclides provide biologically-distributed vehicles for radiotherapy of multifocal cancer. Only an individualized method for prescribing radionuclide dose takes variations in drug pharmacokinetics into consideration, and studies have shown that optimally safe and effective therapy can be best achieved when the prescription is influenced by estimated radiation dose (Flux et al. 2003, DeNardo et al. 2003). The imaging possibilities of MTR are powerful and unique, since they allow the clinician to visualize the distribution of the treatment agent before the treatment. The visualization is achieved by a tracer amount of the radiolabeled drug to be used in the individual patient to determine the pharmacokinetics and subsequently the radiation dose distribution (Siegel et al. 1999, Bolch et al. 1999, Williams 1995). Using the images acquired with the tracer dose, treatment planning in MTR can provide information to the physician to 
(1) qualify the patient for the treatment by estimating the potential benefit and (2) optimize the prescription of targeted radionuclide (Hartmann Siantar et al. 2002). MINERVA provides three-dimensional, patient-specific Monte Carlo based dosimetry for MTR. The advantages of the additional dosimetric accuracy achieved with threedimensional Monte Carlo methods have been described (Johnson and Vessella 1989, Furhang et al. 1997, Furhang et al. 1996, Buffa et al. 2003).

\section{Methods}

\subsection{MINERVA system overview}

The overall MINERVA system is described in detail elsewhere (Lehmann et al. 2004, Wemple et al. under review) and will be introduced here only briefly. MINERVA is a flexible software environment for planning of radiation treatment for multiple modalities with an emphasis on newer forms of radiation therapy and the possibility to create combined plans of different radiation modalities considering biological factors. The system is written in the Java programming language (Gosling et al. 1996). It consists of a program framework with several universal modules, and supports a standardized interface for implementing certain computational components and for extending the capabilities of the application (Application Programming Interface - API). The main modules are the Image module, for import of patient image data in various formats, the Model module, for building a model of the patient based on the images to be used for the calculations, and the Analyze module, for design and analysis of treatment strategies. The actual dose calculating engines are implemented as plugins to allow maximal 
flexibility. The entire MINERVA system is controlled through the Patient module and makes use of a modern database structure. Figure 1 gives an overview of the system.

\subsection{Activity map - MTR Source Module}

MTR treatment planning is accomplished within MINERVA through the use of the MTR Source Module and the MTR Transport plugin. The MTR Source Module offers methods to define the activity map for a targeted radionuclide treatment plan based on patient image information, as described in this section. An explanation of the MTR transportation plugin follows in the next section.

The MTR Source Module currently supports activity map generation in two modes: manual assignment of a constant value to a region and back projected calculation of the average region activity from gamma camera images.

Manual assignment of a constant activity to an organ or any other type of region (specified previously in the Model module) is a simple method that can be used if the more sophisticated methods fail, i.e. due to insufficient image quality or complete lack of radiographic images. The method is also useful as an investigational tool in testing transport codes and comparing the new methods to previously used strategies. Through a menu, an activity can be assigned to any number of regions. The assigned activity can be visualized in an activity view, where grey scale levels correspond to the activity (Figure 2).

Radiographic emission images taken with a tracer amount of radioactivity attached to the drug are used in the back projection method. Back projection also requires the patient geometry information derived from images in the Model module. To employ the method, organs and other regions outlined in the modeling process are 
selected for display in a projected view, which is then used to align the patient geometry with the emission image. At this point, the activity calculation utilizes a single anterior or posterior image of the patient acquired with a gamma camera. This image can either be obtained with a tracer dose of the treatment isotope, as in the case of gamma emitting isotopes (Zelenetz 2003, Wahl 2003) or with a specific imaging surrogate isotope for exclusively beta emitting treatment isotopes (DeNardo et al. 1997).

The graphical user interface provides several tools to translate, scale, and rotate the patient model in order to align it with the radiographic image (Figure 3). To facilitate this process the user has the option to enable or disable the display of specific regions (e.g. organs).

When aligned, each pixel in the image is correlated with a stack of voxels of the patient geometry, defined as the voxels intersected by a ray projected normal to the image plane at that point. In MINERVA the voxels are cubes of identical side-lengths, called univels. Patient images are re-sliced accordingly in the Model module to achieve this geometry (Frandsen et al. 1998).

The calculation of the activity distribution begins with determining the background activity. The user selects a location in the image that is representative of the tissue composition of the background region. The system performs an attenuated backprojection to determine the average activity for the selected location in the image and the 24 nearest neighboring univels. This value is considered the background activity. The user is prompted to select from a checklist which regions in the patient geometry should be considered to contain only background activity. The activity is calculated for all other regions by first subtracting the attenuated background activity and the activity calculated for other relevant regions from the image activity at each pixel. The region 
activity is determined by averaging over all pixels of the region. If the stack of univels contains one or more regions that have no assigned activity, then that stack is not included in the average. Performing this procedure for each region of the patient yields a full three-dimensional activity map of the patient. The user determines the order in which the regions are calculated, and it is advisable to calculate first those regions that have the least overlap with uncalculated regions. The calculated activity map can be visualized in an activity view.

\subsection{Dose calculation in the MTR transport plugin}

The dose calculation for MTR is performed by the PEREGRINE Monte Carlo dose calculation system (Hartmann Siantar et al. 2001). PEREGRINE is configured, invoked and controlled from the MINERVA program framework using a plugin module, as described in section 2.1. The PEREGRINE system is a coupled photon-electron Monte Carlo transport simulator that has been designed to compute dose distributions for external beam radiotherapy. For the application in MINERVA the PEREGRINE code has been extended to include internal photon and electron sources. The code has been successfully tested against published data from other systems. Agreement was found to be generally within $2 \%$ in comparison with EGS4 and within $10 \%$ in comparison with MCNP (Descalle et al. 2003). The transport code is currently being benchmarked against experimental data using an extended liquid source in a solid phantom and incorporating inhomogeneities encountered in the human body such as bones and lungs.

A Java plugin module provides MINERVA users with the seamless capability to invoke the PEREGRINE system for dose calculation from the Analyze module for a 
specific treatment field. The patient data, including the CT image set and the 3D activity distribution, are read in from the database and formatted as input data for the simulation.

Calculation of the dose distribution is made based on a single three-dimensional activity map. The fact that the activity in the patient changes with time can be accounted for with multiple calculations based on activity maps acquired at different time points and appropriate summation of the dose distributions in the analyze module. The way the different time points are combined is intentionally not hard-coded into the system but left to the user since the kinetics are not yet completely understood and are subject to ongoing investigations. MINERVA can therefore be used as a tool in such investigations; dose distributions can be combined in various ways and don't have to be recalculated for different combinations of activity distributions.

The user interface of the plugin allows the user to control simulation parameters such as requested statistical uncertainty or number of histories. The MTR transport plugin manages the run of the Monte Carlo simulation automatically. A typical workflow is illustrated in Figure 4. The plugin, running on the local workstation, retrieves all necessary information from the MINERVA Database (Patient geometry, activity distribution), combines it with the user input simulation parameters, formats the input data and sends a job to the PEREGRINE Queue Database. This database can be local or at a central machine. The connection between the workstation and the database is only necessary for the time of data transmission. One or several machines (PEREGRINE workers) run a script that checks the PEREGRINE Queue Database for waiting jobs in regular intervals ( $30 \mathrm{~s}$ in the current implementation). If a job is waiting in the database, the script on the PEREGRINE worker retrieves the information for the job from the database, executes the simulation according to the given parameters. It provides updates 
on the status of a simulation to the PEREGRINE Queue Database while it is working on a job. After the simulation is finished the script sends the calculated dose distribution back to the database. The user on the local workstation can connect to the database, using the MTR transport plugin, to check on the status of any simulation and retrieve the dose distribution information once it is available. From the local workstation the dose information is then saved in the MINERVA database.

Tools in the Analyze module are available to display the dose distribution overlaid on the CT images or integrated in dose volume histograms (DVH), two common display features in current treatment planning systems.

\section{Results and discussion}

Molecular targeted radiation therapy (MTR) is the first implemented operational capability of the new MINERVA system. Using the plugin principle, an MTR source module and an MTR transport module have been designed, implemented and successfully tested. The Monte Carlo simulations for the dose calculation are run as independent processes with the option of running several of them in parallel on multiple CPUs or machines. Current simulation time for MTR is in the order of several hours to days (depending on desired statistical uncertainty) on of-the-shelf personal computers running the Linux operating system.

The accuracy of the PEREGRINE Monte Carlo simulations for MINERVA has been previously reported on (Descalle et al. 2003). This report includes comparisons of specific absorbed fractions calculated with MINERVA to specific absorbed fractions

published in the revised MIRD Pamphlet 5 (Snyder et al. 1978) following the validation 
approach of Johnson (Johnson et al. 1999). Table 1 shows the results of this comparison for the source organs liver, kidneys and adrenals (100 keV photon sources uniformly distributed) paired with each of the target organs lungs, adrenals, kidneys, liver, pancreas and the spleen in a heterogeneous phantom. The agreement is within $3.7 \%$ for all specific absorbed fractions within the source organs themselves and within 5\% for most others.

The MINERVA MTR implementation has been tested with patient image data. Figures 5 and 6 shows the dose displays from the Analyze module for a Monte Carlo based dose distribution for the treatment isotope I-131. In the MTR source module homogeneous activities have been assigned each to the liver and the kidneys. For experimental purposes the specific activity in the liver was chosen double the specific activity in the kidneys. Figure 5 shows the light box view with coronal cuts. Figure 6 shows an axial slice that contains part of liver and kidneys. It has a legend of the dose level overlaid. For demonstration purposes only 4 dose levels are shown. As expected for the given activity distribution, the dose delivered to the voxels within the liver is approximately double the dose to the voxels within the kidney. The dose is mainly confined to those organs. There are inhomogeneities in the dose distribution within the organs, which are due to statistical uncertainty of the particular simulation, a concern that needs to be addressed for all Monte Carlo simulations. Figure 7 shows the entire user interface of the Analyze module for this I-131 case with a dose-volume-histogram (DVH) tab selected.

While several components of MINERVA are still under development, the successful implementation of MTR calculations is an important step on the way to a system that will incorporate several radiotherapy modalities. MINERVA will enable the 
physician to make clinical decisions based on composite plans with biological weighting factors for the various radiotherapy modalities. It will assist researchers with tools to identify these factors.

MINERVA is open for extension by users through the API. Several groups have already indicated strong interest to work with the code. The next step underway at INEEL and MSU is the implementation of the neutron transport plugin. External beam photon and electron capabilities using widely available codes are to follow.

\section{Acknowledgements}

This work was performed under the auspices of the U.S. Department of Energy, Office of Science, by University of California Lawrence Livermore National Laboratory under contract No. W-7405-Eng-48 and by the Idaho National Engineering and Environmental Laboratory under contract No. DE-AC07-99ID13727.

\section{References}

Benua, R S, Cicale, N R, Sonenberg, M and Rawson, R W 1962 The relation of radioiodine dosimetry to results and complications in the treatment of metastatic thyroid cancer Am J Roentgenol Radium Ther Nucl Med 87 171-82

Bodey, R K, Flux, G D and Evans, P M 2003 Combining dosimetry for targeted radionuclide and external beam therapies using the biologically effective dose Cancer Biother Radiopharm 18 89-97 
Bolch, W E, Bouchet, L G, Robertson, J S, Wessels, B W, Siegel, J A, Howell, R W, Erdi, A K, Aydogan, B, Costes, S, Watson, E E, Brill, A B, Charkes, N D, Fisher, D R, Hays, M T and Thomas, S R 1999 MIRD pamphlet No. 17: the dosimetry of nonuniform activity distributions--radionuclide $S$ values at the voxel level. Medical Internal Radiation Dose Committee J Nucl Med 40 11S-36S

Buffa, F M, Verhaegen, F, Flux, G D and Dearnaley, D P 2003 A Monte-Carlo method for interface dosimetry of beta emitters Cancer Biother Radiopharm 18 463-71

DeNardo, G, Yuan, A, Goldstein, D, Richman, C, O'Donnell, R, Shen, S, Hartmann Siantar, C and DeNardo, S 2003 Impact of interpatient pharmacokinetic variability on design considerations for therapy with radiolabeled MAbs Cancer Biother Radiopharm 18 231-7

DeNardo, G L, Raventos, A, Hines, H H, Scheibe, P O, Macey, D J, Hays, M T and DeNardo, S J 1985 Requirements for a treatment planning system for radioimmunotherapy Int J Radiat Oncol Biol Phys 11 335-48

DeNardo, S J, Richman, C M, Goldstein, D S, Shen, S, Salako, Q, Kukis, D L, Meares, C F, Yuan, A, Welborn, J L and Denardo, G L 1997 Yttrium-90/indium-111DOTA-peptide-chimeric L6: pharmacokinetics, dosimetry and initial results in patients with incurable breast cancer Anticancer Res 17 1735-44

Descalle, M A, Hartmann Siantar, C L, Dauffy, L, Nigg, D W, Wemple, C E, Yuan, A and DeNardo, G L 2003 Application of MINERVA Monte Carlo simulations to targeted radionuclide therapy Cancer Biother Radiopharm 18 71-9

Flux, G D, Guy, M J, Beddows, R, Pryor, M and Flower, M A 2002 Estimation and implications of random errors in whole-body dosimetry for targeted radionuclide therapy Phys Med Biol 47 3211-23 
Flux, G D, Guy, M J, Papavasileiou, P, South, C, Chittenden, S J, Flower, M A and Meller, S T 2003 Absorbed dose ratios for repeated therapy of neuroblastoma with I-131 mIBG Cancer Biother Radiopharm 18 81-7

Frandsen, M W, Wessol, D E, Wheeler, F J and Starkey, D 1998 In Eighth International Symposium on Neutron Capture TherapyPlenum Press, New York, La Jolla, CA, USA

Furhang, E E, Chui, C S, Kolbert, K S, Larson, S M and Sgouros, G 1997 Implementation of a Monte Carlo dosimetry method for patient-specific internal emitter therapy Med Phys 24 1163-72

Furhang, E E, Chui, C S and Sgouros, G 1996 A Monte Carlo approach to patientspecific dosimetry Med Phys 23 1523-9

Gosling, J, Joy, B and Steele, G 1996 The Java Language Specification Addison-Wesley Hartmann Siantar, C L, Vetter, K, DeNardo, G L and DeNardo, S J 2002 Treatment planning for molecular targeted radionuclide therapy Cancer Biother Radiopharm $17267-80$

Hartmann Siantar, C L, Walling, R S, Daly, T P, Faddegon, B, Albright, N, Bergstrom, P, Bielajew, A F, Chuang, C, Garrett, D, House, R K, Knapp, D, Wieczorek, D J and Verhey, L J 2001 Description and dosimetric verification of the PEREGRINE Monte Carlo dose calculation system for photon beams incident on a water phantom Med Phys 28 1322-37

Johnson, T K, McClure, D and McCourt, S 1999 MABDOSE. II: Validation of a general purpose dose estimation code Med Phys 26 1396-403 
Johnson, T K and Vessella, R L 1989 On the possibility of 'real-time' Monte Carlo calculations for the estimation of absorbed dose in radioimmunotherapy Comput Methods Programs Biomed 29 205-10

Lehmann, J, Hartmann Siantar, C L, Wessol, D E, Wemple, C A, Nigg, D W, Cogliati, J J, Daly, T P, Descalle, M A, Flickinger, T, Pletcher, D and DeNardo, G L 2004 Monte Carlo Treatment Planning for Molecular Targeted Radiotherapy within the MINERVA system Current Topics in Monte Carlo Treatment Planning, Advanced Workshop, May 3 - 5, 2004 at McGill University, Montreal, Canada, oral presentation

Nigg, D W 2003 Computational dosimetry and treatment planning considerations for neutron capture therapy J Neurooncol 62 75-86

Nigg, D W, Wheeler, F J, Wessol, D E, Capala, J and Chadha, M 1997 Computational dosimetry and treatment planning for boron neutron capture therapy J Neurooncol 33 93-104

Rubin, P 1989 Law and order of radiation sensitivity. Absolute versus relative Front Radiat Ther Oncol 23 7-40

Siegel, J A, Thomas, S R, Stubbs, J B, Stabin, M G, Hays, M T, Koral, K F, Robertson, J S, Howell, R W, Wessels, B W, Fisher, D R, Weber, D A and Brill, A B 1999 MIRD pamphlet no. 16: Techniques for quantitative radiopharmaceutical biodistribution data acquisition and analysis for use in human radiation dose estimates $J$ Nucl Med 40 37S-61S

Snyder, W S, Ford, M R, Warner, G G and Watson, S B 1978 Estimates of absorbed fractions for monoenergetic photon sources uniformly distributed in various 
organs of a heterogeneous phantom - Revised MIRD pamphlet No. 5 Society of Nuclear Medicine

Stabin, M G 1999 Internal dosimetry in the use of radiopharmaceuticals in therapy-science at a crossroads? Cancer Biother Radiopharm 14 81-9

Thomas, S R 2002 Options for radionuclide therapy: from fixed activity to patientspecific treatment planning Cancer Biother Radiopharm 17 71-82

Wahl, R L 2003 The clinical importance of dosimetry in radioimmunotherapy with tositumomab and iodine I 131 tositumomab Semin Oncol 30 31-8

Wemple, C A, Wessol, D E, Nigg, D W, Cogliati, J J, Milvich, M, Fredrickson, C M, Perkins, M, Harkin, G J, Hartmann Siantar, C L, Lehmann, J, Flickinger, T, Pletcher, D, Yuan, A and DeNardo, G L under review MINERVA - A Multimodal Radiation Treatment Planning System Radiation Protection Dosimetry

Williams, L E 1995 Estimation of absorbed doses in radioimmunotherapy Med Phys 22 958

Zelenetz, A D 2003 A clinical and scientific overview of tositumomab and iodine I 131 tositumomab Semin Oncol 30 22-30 


\section{Tables}

Table 1. Comparison of specific absorbed fractions calculated with the MINERVA code and the specific absorbed fractions given in revised MIRD pamphlet 5 (Snyder et al. 1978) for $100 \mathrm{keV}$ photons emitted by source organs of an heterogeneous phantom.

Source organs are the adrenals, the kidneys and the liver, and target organs are the lungs, adrenals, kidneys, liver, pancreas and spleen. (Table partially adapted with permission from (Descalle et al. 2003))

\begin{tabular}{|c|c|c|c|}
\hline \multirow{2}{*}{ Target organs } & \multicolumn{3}{|c|}{ Relative difference in absorbed fraction MIRD 5 / MINERVA [\%] } \\
& Adrenals & $\begin{array}{c}\text { Source organs } \\
\text { Kidneys }\end{array}$ & Liver \\
\hline Lungs & -11.9 & 0.8 & 3.5 \\
Adrenals & -3.7 & 7.1 & -23.4 \\
Kidneys & 5.7 & 1.0 & 4.0 \\
Liver & 3.4 & -0.9 & -1.6 \\
Pancreas & 5.0 & -1.5 & -0.5 \\
Spleen & -2.3 & -3.6 & -3.9 \\
\hline
\end{tabular}




\section{Figure captions}

Figure1

Overview of the MINERVA system. Reprint from (Wemple et al. under review)

Figure 2

Activity view in the MTR source module. A homogeneous activity has been assigned to liver and both kidneys. Grey level corresponds to activity. The pointer is over the liver and activity (100.0 microCi/m) is displayed in lower left corner.

Figure 3

Image view in the MTR source module. The outlined patient organs are aligned with the emission image and the imaging isotope is specified.

Figure 4

Workflow of the MTR transport plugin. The plugin retrieves all necessary information from the MINERVA Database, combines it with the user input simulation parameters, and sends a job to the PEREGRINE Queue Database. A script on a PEREGRINE workers checks the database for waiting jobs in regular intervals, retrieves the information for the job from the database, executes the simulation according to the given parameters and sends the calculated dose distribution back to the database. The user on the local workstation can connect to the database through the MTR transport plugin to check on the status of any simulation and retrieve the dose distribution information once it is available. From the local workstation the dose information is then saved in the MINERVA database. 
Figure 5

Dose display for an MTR plan. Monte Carlo based dose distribution for an MTR with the isotope I-131. Light box view with coronal cuts.

\section{Figure 6}

Dose display for an MTR plan. Monte Carlo based dose distribution for an MTR with the isotope I-131. The axial view with legend of the dose level overlaid. For demonstration purposes only 4 dose levels are shown.

\section{Figure 7}

Dose display for an MTR plan. Monte Carlo based dose distribution for an MTR with the isotope I-131. Sample dose volume histograms for liver, kidneys and stomach are shown within the user interface of the Analyze module. 


\section{Monte Carlo Treatment Planning for Molecular Targeted}

\section{Radiotherapy within the MINERVA system}

Figure 1

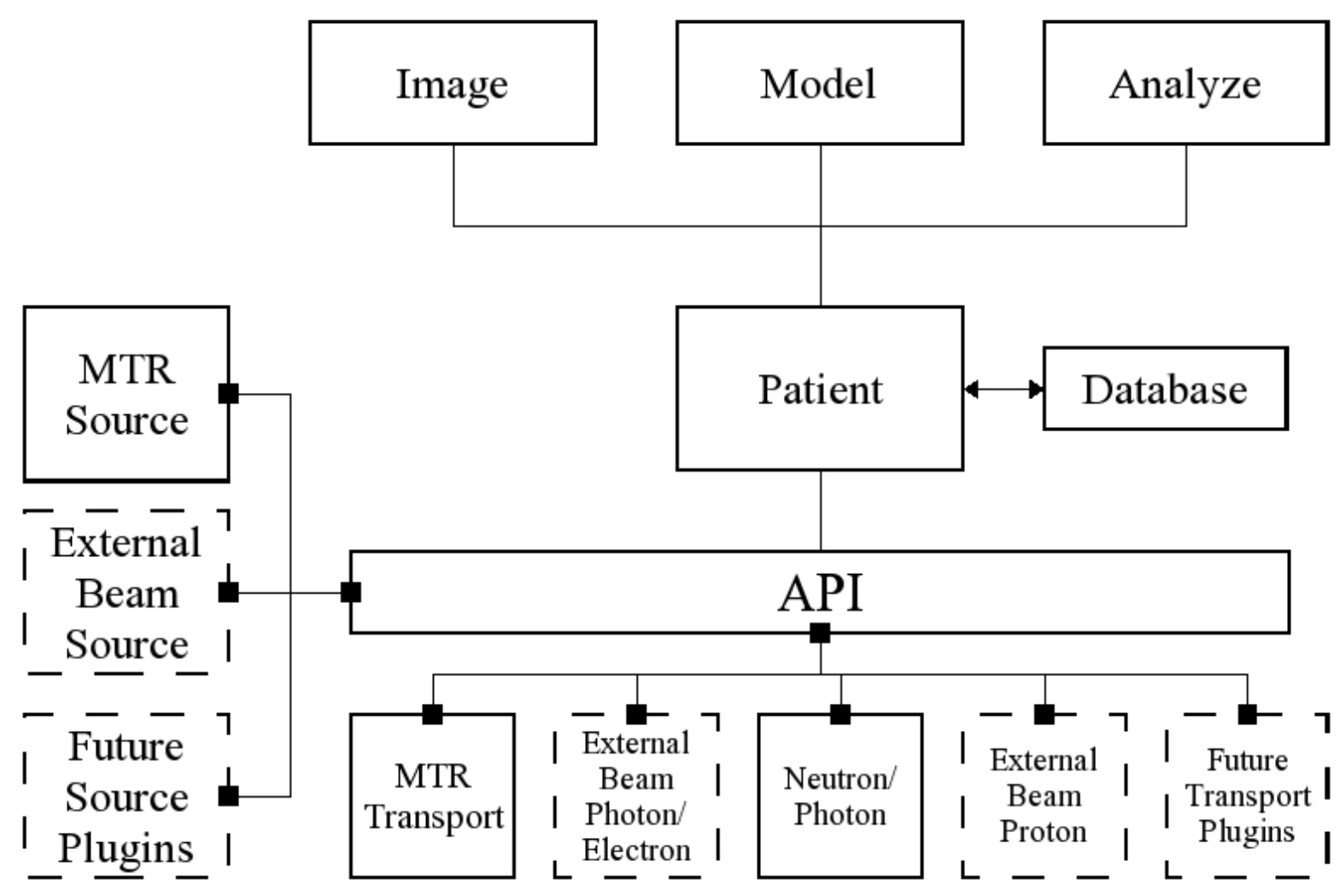


Figure 2

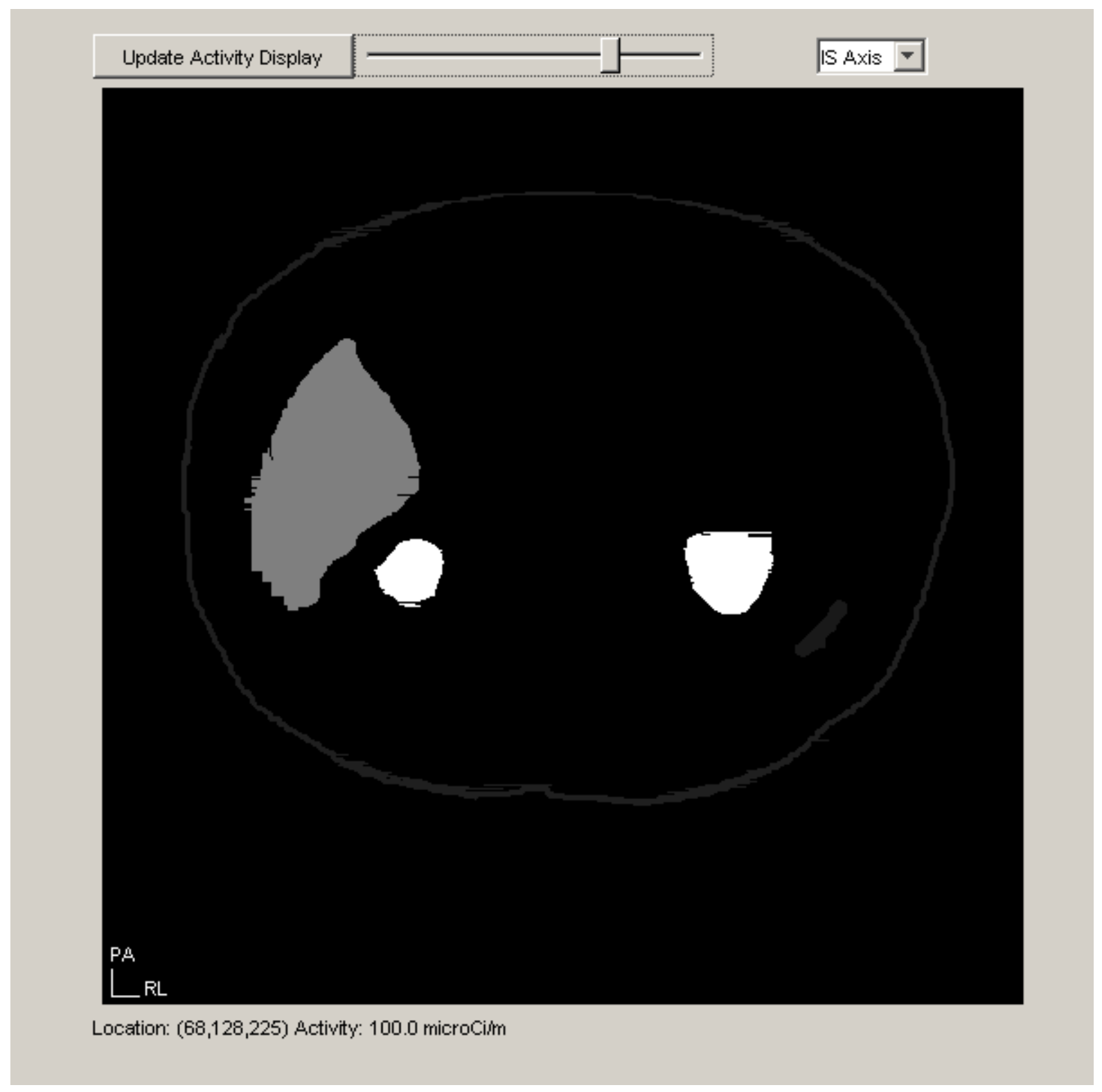


Figure 3

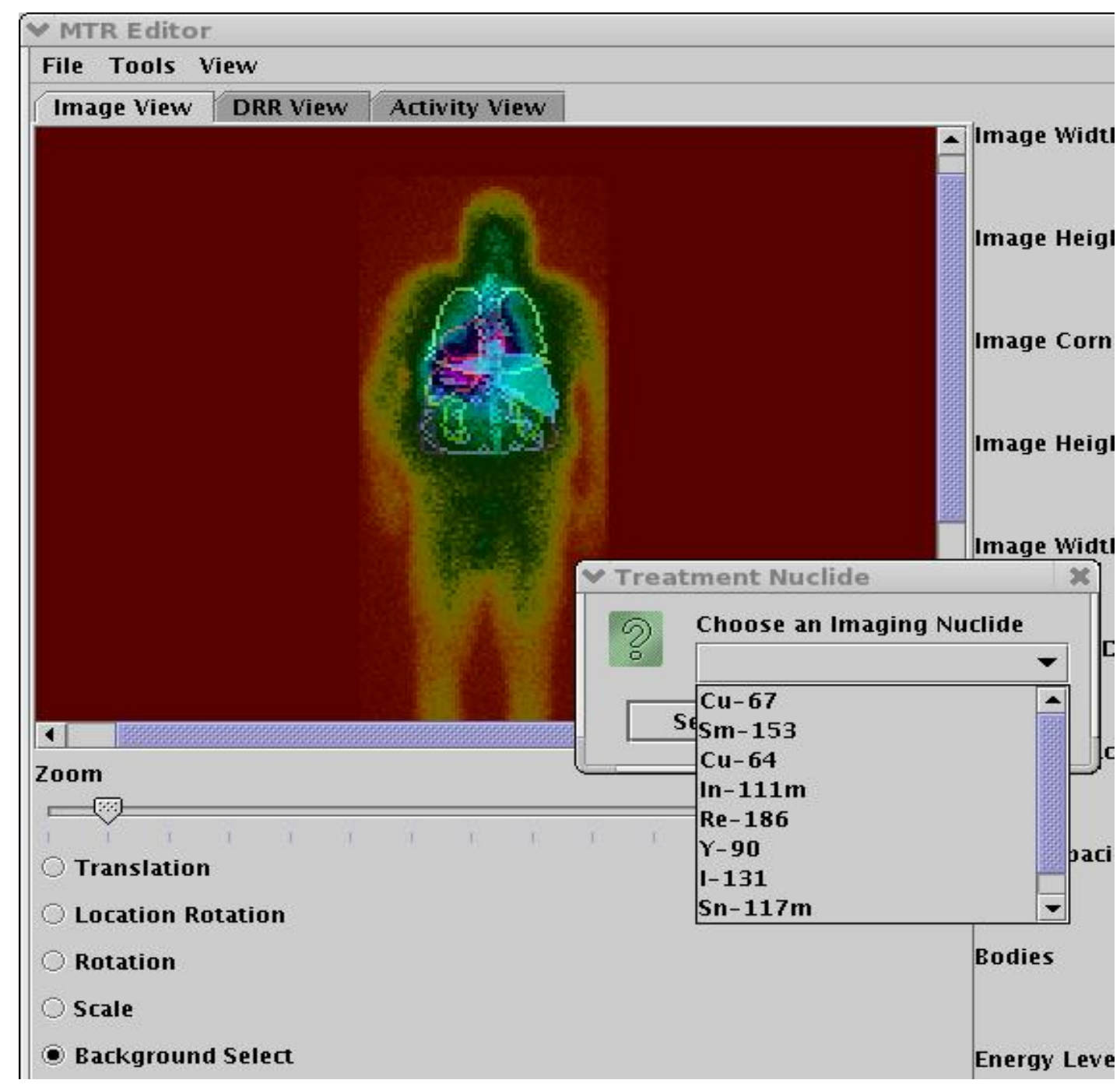




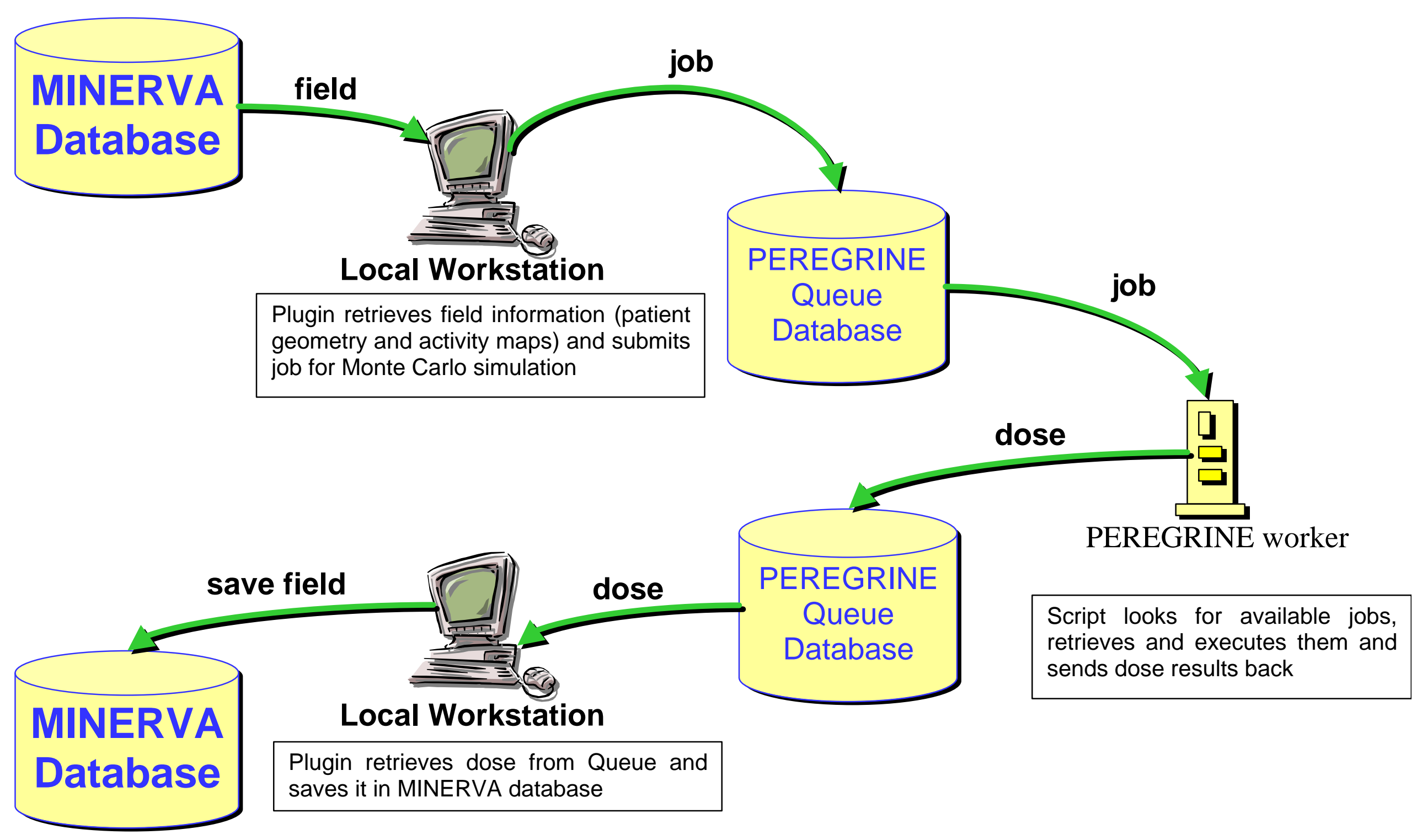


Figure 5

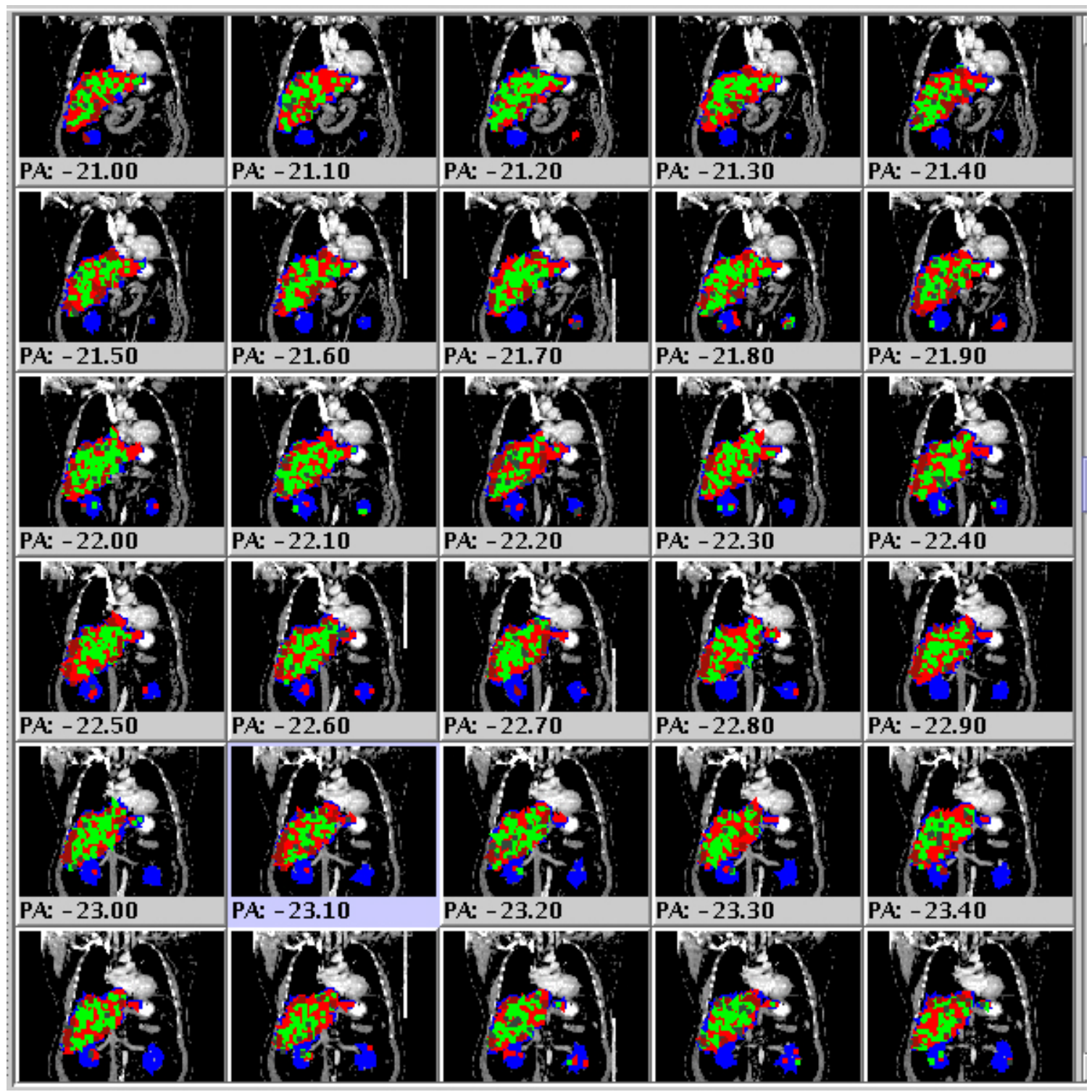


Figure 6

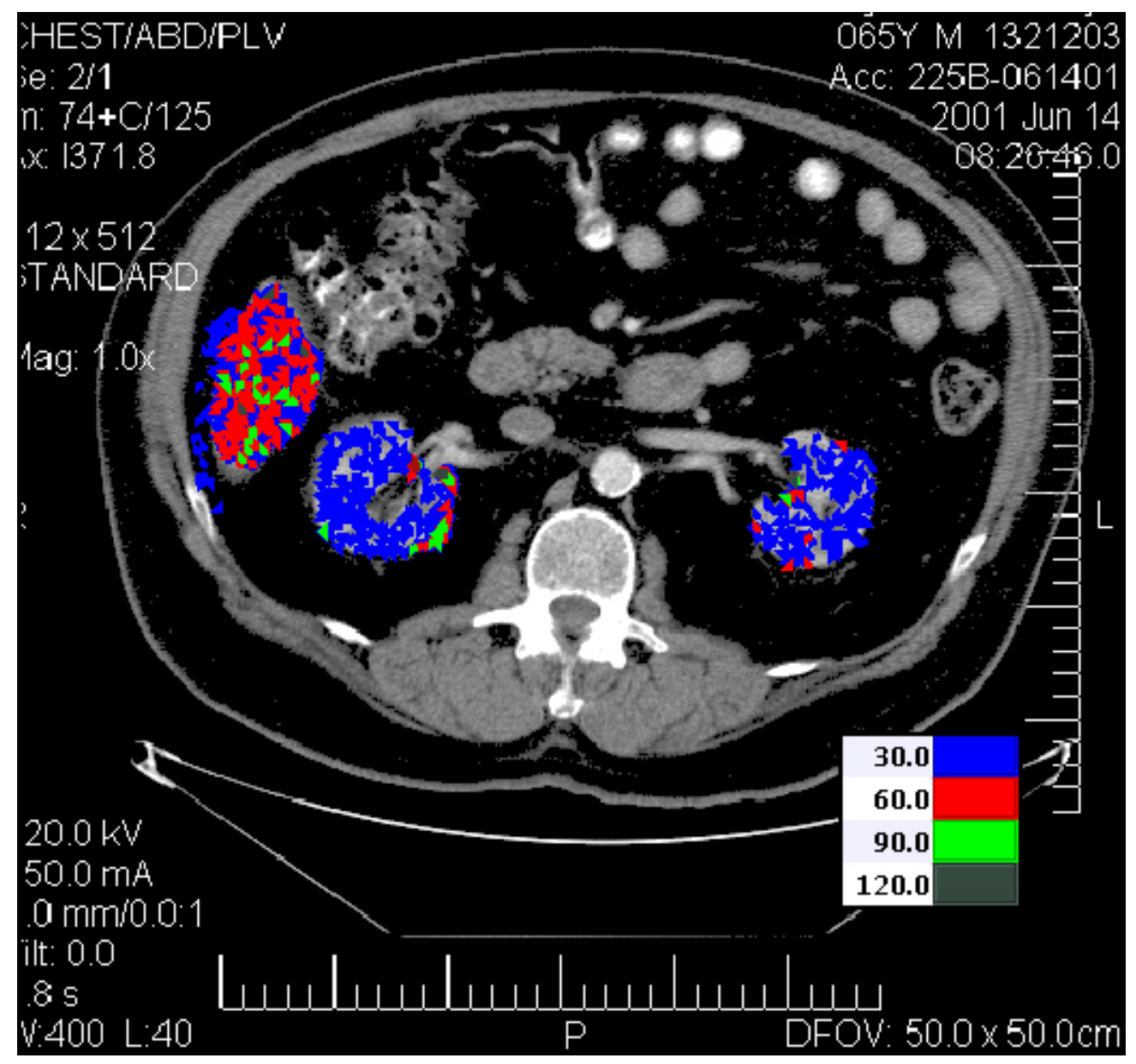




\section{Figure 7}

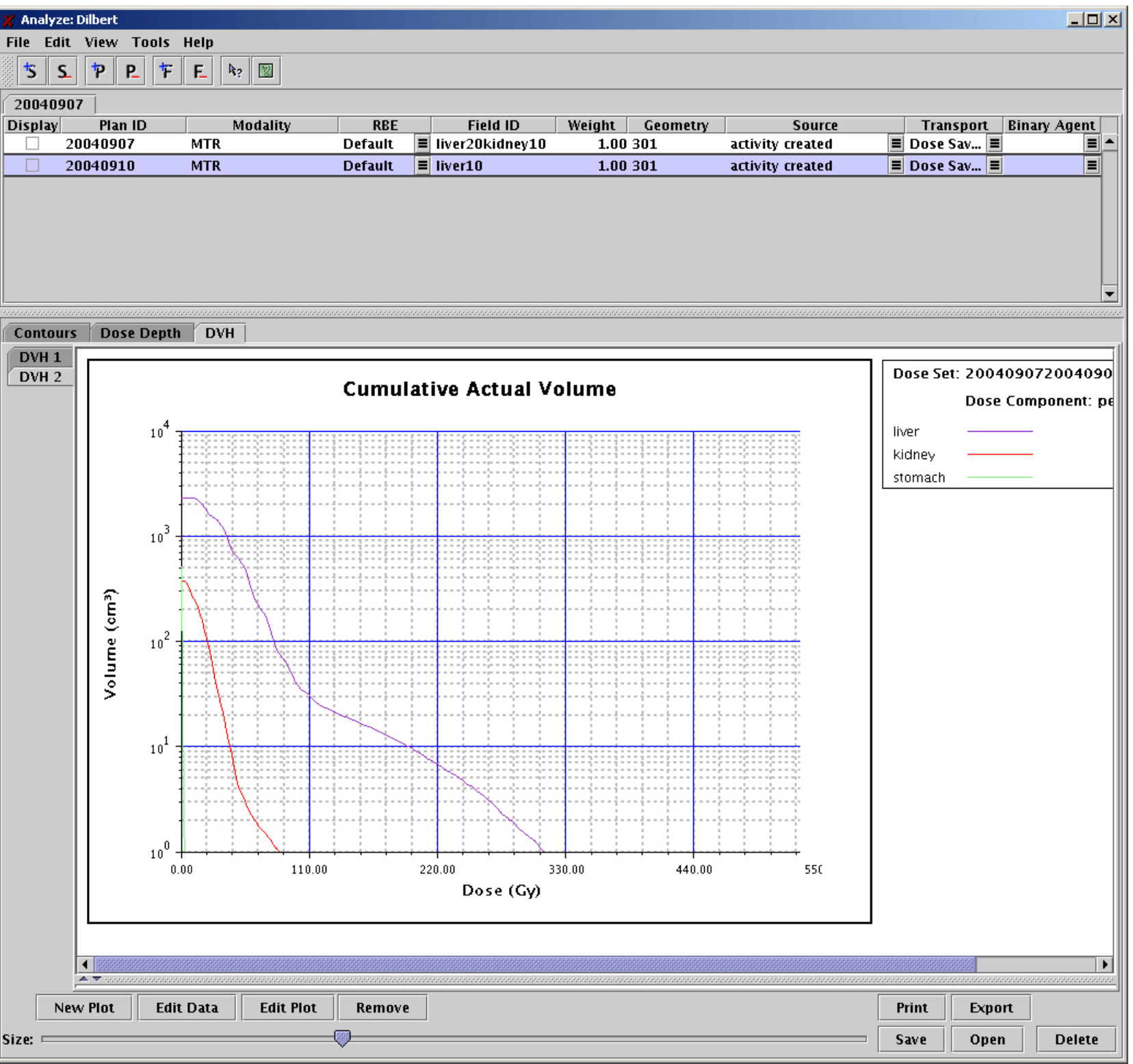

